\title{
Effects of Reference Axes Used During Measurements of Ocular and Corneal Higher-Order Aberrations in Patients Following LASIK
}

\author{
Atsuko Takehara ${ }^{1}$, Naoyuki Maeda ${ }^{1}$, Sayuri Ninomiya ${ }^{2}$, Takashi Fujikado ${ }^{2}$, \\ Yoko Hirohara ${ }^{3}$, and Toshifumi Mihashi ${ }^{3}$ \\ ${ }^{1}$ Department of Ophthalmology, Osaka University Medical School, Suita, Osaka, Japan; \\ ${ }^{2}$ Department of Applied Visual Science, Osaka University Medical School, Suita, Osaka, Japan; \\ ${ }^{3}$ Topcon Corporation, Tokyo, Japan
}

Japanese Journal of Ophthalmology 2006;50:318-322

The correct name of the fifth author should be given as Yoko Hirohara, not Yoko Hiroha.

\footnotetext{
Received: July 1, 2005 / Accepted: November 2, 2005

Correspondence and reprint requests to: Naoyuki Maeda, Room E7, Department of Ophthalmology, Osaka University Medical School, Yamadaoka 2-2, Suita, 565-0871, Japan

e-mail:nmaeda@ophthal.med.osaka-u.ac.jp

Presented in part as a poster at the Association of Research for Vision and Ophthalmology annual meeting, Fort Lauderdale, Florida, May 6, 2003. Mr. Mihashi and Ms. Hirohara are employees of Topcon Corporation. No other author or family member has any proprietary or financial interest in the instrument used in the study.
} 